\title{
Activation of Embryonic/germ Cell-like Axis Links Poor Outcomes of Gliomas
}

\author{
Zhan Ma \\ Huashan Hospital, Fudan University \\ Fengyu Zhang \\ Huashan Hospital, Fudan University \\ Haishi Zhang \\ Huashan Hospital, Fudan University \\ Hui-Kuan Lin \\ Wake Forest Baptist Medical Center \\ chunfang liu ( $\nabla$ chunfang_liu@fudan.edu.cn ) \\ Huashan Hospital, Fudan University
}

\section{Research Article}

Keywords: Gametogenesis hypothesis of tumours, Glioma prognosis, Embryonic/germ cell-like cycle, Primordial germ cell-like tumor cells, Markers for poor outcomes of glioma patients

Posted Date: July 26th, 2021

DOl: https://doi.org/10.21203/rs.3.rs-550958/v1

License: (c) (1) This work is licensed under a Creative Commons Attribution 4.0 International License. Read Full License 


\section{Abstract}

It is unclear which core events drive the malignant progression of gliomas. Earlier studies have revealed that the embryonic stem (ES) cell/early PGC state is associated with tumourigenicity. Here, our findings showed that germ cell-like cells were present in human gliomas and cultured glioma cells and that the formation of germ cell-like cells was essential for glioma tumours. Bioinformatic analysis showed that the mRNA levels of genes related to embryonic/germ cell development could be detected in most gliomas. Our findings showed that the activation of genes related to reprogramming or the germ cell-like state alone seemed to be insufficient to lead to a malignant prognosis, whereas increased mRNA levels of genes related to the activation of the embryonic/germ cell-like cycle (somatic PGC-EGC-like cycle and somatic parthenogenetic embryo-like cycle) were positively correlated with malignant prognoses and poor clinical outcomes of gliomas. Genes related to the embryonic/germ cell cycle alone or in combination with the WHO grade or $1 \mathrm{p} 19 \mathrm{q}$ codeletion status could be used to subdivide gliomas with distinct clinical behaviours. Together, our findings indicated that a crucial role of germ cell-like cell formation in glioma initiation as well as activation of genes related with the parthenogenetic embryo-like cycle and PGC-EGC-like cycle link to the malignant prognosis and poor outcomes of gliomas, which might provide a novel way to better understand the nature of and develop targeted therapies for gliomas as well as important markers for predicting clinical outcomes in gliomas.

\section{Introduction}

Despite differences in their tissues of origin and genetic backgrounds, many tumours exhibit common phenotypes, such as high embryonic/germ cell traits, which is accounted for in the embryo/gametogenesis-related hypothesis of tumours proposed by Müller (1838), Langenbeck (1840) and Beard (1902) and extended by Old (2001)[1-5]. This hypothesis postulated that tumours arise from germ cells or reactivation of the germ cell programme in somatic tissues. Earlier studies have revealed that tumourigenicity is an inherent feature of the embryonic stem (ES) cell/early primordial germ cell (PGC) state, including early PGCs, implantation embryos, parthenogenetic oocytes, embryonal carcinoma (EC) cells, embryonic stem (ES) cells, embryonic germ cells (EGCs) and induced pluripotent stem (iPS) cells [6-11], raising the possibility that re-obtaining the ES/early PGC state may be one of the driving events in the malignant behaviours of somatic tumours.

An increasing number of studies have revealed that embryonic/germ cell-specific genes play crucial roles in tumourigenicity, metastasis and therapy resistance [12-25]. Notably, it has been shown that knockout of those genes related to germ cell development fully inhibits brain tumour formation in Drosophila [26], and no melanoma initiation occurs before the reactivation of genes related to embryonic development in a zebrafish model [27]. Our previous studies showed that embryonic/germ cell-like tumour cells were found in various types of tumours, played important roles in tumour growth, liver metastasis and drug resistance; and exhibited an independent life cycle [19-21, 28-31]. We also showed that the formation of embryonic/germ cell-like tumour cells was induced by either p53 deficiency or a chemical carcinogen (3methylcholanthrene, 3-MCA) $[20,28]$. In essence, PGCs give rise to sperm or oocytes and then return to 
the embryonic state via fertilization or parthenogenesis. However, PGCs can also return to the embryonic state via PGC-EGC conversion under some conditions, such as PTEN/TP53/SOX17 deficiency [9, 32, 33]. Therefore, we postulated that the acquisition and maintenance of the ES/early PGC state via activation of the embryonic/germ cell-like developmental axis might be linked to the core malignant behaviours of somatic tumours through somatic cell-ESC/PGC-(post-migratory PGC)-EGC-somatic cell-like conversion (which we named the somatic PGC-EGC/ES-like cycle) and/or somatic tumour cell-ESC/PGC-(postmigratory PGC)-oocyte-parthenogenetic embryo-somatic tumour cell conversion (which we named the somatic parthenogenetic embryo-like cycle) [20] (Fig. 1A). Here, our findings showed that activation of the embryonic/germ cell-like development axis was essential in the malignant progression of gliomas and that related genes could be used as important markers to predict clinical outcomes in gliomas.

\section{Methods}

\section{Ethics statement}

The study protocol was approved by the Medical Ethics Committee of Huashan Hospital, Fudan University (permit number MEC-HS (Hu) 2011-362), and informed consent was obtained from each patient. All research was performed in accordance with relevant guidelines and regulations. Our study was carried out in compliance with the American Veterinary Medical Association (AVMA) Guidelines for the Euthanasia of Animals (2020). All animal protocols were carried out in accordance with ARRIVE guidelines. All animal experiments were approved by with the Institutional Animal Care and Use Committee of Fudan University (approval number: 2019JS-073).

\section{Cell Culture}

U251, LN229 and A172 ${ }^{\text {wt }}$ cells were obtained from ATCC. A172 ${ }^{\text {mut }}$ cells were derived from A172 ${ }^{\text {wt }}$ cells after treatment with a chemical carcinogen (3-methylcholanthrene, 3-MCA). All cells were cultured in highglucose Dulbecco's modified Eagle's medium (DMEM, HyClone) with $10 \%$ foetal bovine serum (FBS; Sigma) and $1 \% \mathrm{~L}$-glutamine and were maintained at $37^{\circ} \mathrm{C}$ with $5 \% \mathrm{CO}_{2}$.

\section{Single cell cloning}

U251 or $\mathrm{A} 172^{\text {mut }}$ cells were plated in 96-well plates by the limited dilution method and incubated at $37^{\circ} \mathrm{C}$ with $5 \% \mathrm{CO}_{2}$ for proliferation. Wells with single cells were selected and then treated with AP staining after culturing for 3 weeks. The efficiency of generating PGC-like cells was counted in the single clones.

\section{Alkaline Phosphatase Staining}


Cultured cells were fixed with $4 \%$ paraformaldehyde in PBS for 4 min, washed twice with a Tris- $\mathrm{HCl}$ $(\mathrm{pH}=8.6)$ buffer solution and then incubated with AP substrate (Vector laboratory) for $40 \mathrm{~min}$ at room temperature.

\section{Real-time PCR analysis}

RNA was isolated from cultured cells by Trizol reagent (Invitrogen) and then converted to cDNA by reverse transcription using a reverse transcription kit (Invitrogen). Real-time PCR analysis was performed with a SYBR Green PCR Master Mix Kit (Applied Biosystems) according to the manufacturer's instructions.

\section{Animal experiments}

All animal protocols were carried out in accordance with ARRIVE guidelines.

To compare tumorigenicity, the U251, LN229, A172 ${ }^{\text {wt }}$ and A172 ${ }^{\text {mut }}$ cells, U251-DAZL ${ }^{+/}$, LN229-DAZL ${ }^{+/}$, A172mut-DAZL ${ }^{+/}$cells, U251-guide, LN229-guide and A172 ${ }^{\text {mut }}$-guide $\left(1 \times 10^{6}\right.$ cells/mice) were subcutaneously injected into female nude mice $(n=5$, age 6 weeks, body weight 20-22 g) respectively. The time of tumour initiation was recorded every day. Five mice were raised in a cage under specific pathogen-free conditions and a 12-h light/dark cycle at $23 \pm 2{ }^{\circ} \mathrm{C}$ and $60 \pm 10 \%$ humidity, and standard food and water were freely available. At the end of the experiment, the mice were euthanized via exposure to a gradually increasing concentration of carbon dioxide gas in accordance to ARRIVE guidelines and then resected the subcutaneous tumors which were then fixed in $4 \%$ formaldehyde for pathological analysis. A P-value $<0.05$ was considered to be significant.

\section{Immunochemistry}

Cells were cultured on coverslips. fixed with $4 \%$ paraformaldehyde and then blocked in PBS with $5 \%$ BSA and $0.05 \%$ Triton-X-100. Cells or tissue sections were incubated overnight at $4{ }^{\circ} \mathrm{C}$ with various primary antibodies, including anti-POU5F1 (ab184665, Abcam), anti-Sox2 (MAB2018R-100, R\&D), anti-Nanog (NB100-58842, Novus Biologicals), anti-Nanos3 (ab70001, Abcam), anti-DDX4 (ab27591, Abcam), or antiDAZL (NB100-2437, Novus biologicals). Next, the cells were stained with Cy3 fluorescent dye-conjugated secondary antibodies (Jackson) and 4,6-diamidino-2-phenylindole (DAPI; Invitrogen), while the tissue sections were stained with haematoxylin.

\section{DAZL deletion}

The CRISPR/Cas9 and sgRNA-RFP plasmids were provided by Addgene and Sigma, respectively [24]. The DAZL-sgRNAs sequences are: GAAGCTTCTTTGCTAGATATGG (Table S1). The guide empty vector was 
used as the blank control (Sigma). To delete DAZL in U251, LN229 and A172 ${ }^{\text {mut }}$ cells, we transfected glioma cells simultaneously with the CRISPR/Cas9 vector and guide RNAs (gRNAs) as described previously [21, 24]. Guide gene was used as a knockout control. Two days post transfection, the treated glioma cells were selected with $4 \mu \mathrm{g} / \mathrm{ml}$ of blasticidin or $5 \mu \mathrm{g} / \mathrm{ml}$ of puromycin for 5 days. Single cells were then isolated from the selected cells by dilution and clonally expanded. Single clone cells with DAZL deletion were selected by gene sequencing.

\section{Tissue specimens}

Glioma tissues were obtained from 38 patients with gliomas, including 20 grade IV gliomas, 8 grade III gliomas and 10 grade II gliomas. All patients underwent curative resection at Huashan Hospital of Fudan University from 2011 to 2013. Glioma tissues were stained with hematoxylin eosin (HE) or antibody.

\section{Data of the CGGA database}

Data from the Cancer Genome Atlas (TCGA), the Genotype-Tissue Expression (GTEx) and the Chinese Glioma Genome Atlas (CGGA) (http://cgga.org.cn/index.jsp) were used to confirm our concept. The Data from the Cancer Genome Atlas (TCGA) and the Genotype-Tissue Expression (GTEx) were analysed using the http://gepia2.cancer-pku.cn/\#analysis. The WEseq_286 dataset was used to analyse the genetic mutations. The methyl_159 dataset was used to analyse the methylation status of various genes. The mRNAseq_325 and mRNAseq_693 datasets (Table S2) were used to analyse the mRNA levels of various genes as well as to investigate whether the mRNA level was associated with the pathologic grades or outcomes of gliomas. The mRNA-array_301 dataset was used to analyse the mRNA level of DAZL in gliomas and its link to the pathologic grades and outcomes of gliomas. Data without overall survival information were deleted. Detailed information on the datasets for mRNA sequencing is provided in Table S2 in Supplementary Appendix.

\section{Statistical analysis}

Kaplan-Meier curves were used to assess overall survival for each of the genes and test groups. Unpaired $t$ test with Welch's correction and Mann Whitney test were used to analyse the difference of gene expression between distinct groups.

\section{Results}

\section{A crucial role of germ cell-like cell formation in tumour initiation}


PGC-like cells were frequently observed in U251 and A172 mutant (A172 ${ }^{\text {mut }}$ ) glioma cell cultures but were barely observed in L229 and A172 wild-type (A172 wt) glioma cell cultures (Fig. 1B-D). We found that germ cell-like cell formation was positively correlated with tumour initiation in glioma cells, including U251, LN229, A172 ${ }^{\text {wt }}$ and A172 ${ }^{\text {mut }}$ cells (Fig. 1D and E). Notably, A172 ${ }^{\text {wt }}$ cells without germ cell-like cell properties failed to form tumour within 120 days after grafting in nude mice. However, $A 172$ mut cells that reobtained germ cell-like properties after treatment with a chemical carcinogen (3-methylcholanthrene, 3MCA) gave rise to tumours within 2 weeks of grafting in nude mice (Fig. 1E). Interestingly, tumour tissues derived from A172 mut cells were teratocarcinomas (Fig. 1F), which provided strong support for the presence of germ cell-like cells since teratocarcinomas are thought to originate from germ cells $[2,5]$. Germ cell-like tumour cells often grow above somatic tumour cells and can be easily separated from somatic tumour cells in vitro. We separated the upper cultures, which were enriched with PGClike cells, from the bottom cultures, which contained a small proportion of PGC-like cells in A172mut cells, by shaking the flask rigorously. RT-PCR data showed that the expression of genes related to germ cells was much higher in the upper cells than in the bottom cells in A172 mut cultures (Fig. 1G). Compared to the bottom cells, the upper cells in A172 ${ }^{\text {mut }}$ cultures showed increased tumour initiation abilities after injection into nude mice (Fig. 1G). After culture, most single U251 and A172 mut cells generated clones containing PGC-like cells, indicating that PGC-like cells could be derived from somatic glioma cells (Fig. 1H).

We then investigated the relationship between germ cell formation and tumour initiation by deleting DAZL, a gene related to the maintenance of PGC fate, with CRISPR-Cas9 technology. Since DAZL ${ }^{-/-}$

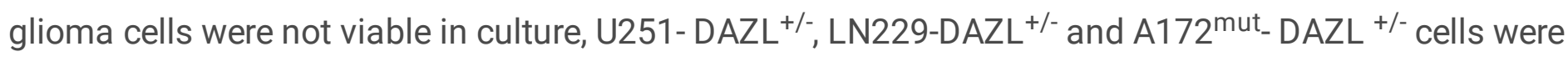
studied (Table S2). Our earlier study revealed that knockdown of DAZL inhibited tumour formation and increased the therapeutic sensitivity of cultured human glioma cells ${ }^{24}$. In this study, we further revealed that knockdown of DAZL greatly impaired germ cell formation and tumour initiation in U251, LN229 and A172 ${ }^{\text {mut }}$ cells (Fig. $1 \mathrm{I}$ and $\mathrm{J}$ ). These findings showed that activation of PGC-like cell formation from somatic tumour cells was essential in the initiation of glioma cell tumours, leading to tumourigenicity in new sites. Thus, activation of the PGC-like state might be necessary for a more aggressive stage of gliomas.

\section{Genetic and epigenetic changes in genes related to embryonic/germ cell development in gliomas}

PGC specification arises from ES cells [34-36], and findings in iPS cells highlight the possibility of somatic cell reprogramming [11]. This means that if a PGC-like fate truly occurs in gliomas, at least two key events are possibly involved: reprogramming and PGC specification (Fig. 1A). Therefore, we investigated whether the genes related to reprogramming, PGC specification and PGC development (including conversion of PGCs into EGCs and oogenesis from PGCs) were activated in gliomas and linked to the malignant prognosis of gliomas (Fig. 1A). The defined gene groups included those 
involved in reprogramming (inhibition: TP53; promotion: POU5F1, SOX2, MYC, KLF4), pluripotency (POU5F1, SOX2, MYC, KLF4, NOTCH1), induction of ES-PGC conversion by microenvironment (BMP2, BMP4, BMP8B, LIF), PGC specification (POUF51, PRDM1, SOX17, ACVR1, IFITM3), PGC fate maintenance (SOX17, PRDM1, KIT, Nanos3, DND1), PGC survival (DAZL, DDX4), proliferation/migration/survival (ITGB1, CXCR4, WNT5A, ROR2), meiosis (SYCP3, DMC1) , PGC-EGC conversion (inhibition: TP53, PTEN, BMP2, BMP4, SOX17; promotion: LIF, STAT3), oocytes (ZP3, GDF15) as well as early embryos (GDF15) [11, 33-35, 37-42].

To validate our hypothesis, Chinese Glioblastoma Genome Atlas (CGGA) data were analysed. Since tumourigenicity is thought to be the outcome of a series of genetic and epigenetic changes, we first investigated whether genetic and epigenetic changes in the gene groups were present in human gliomas. The whole-exome sequencing results of gliomas from the CGGA database showed that there were few or no genetic changes among a series of core genes and signalling pathways related to the embryonic/germ cell developmental axis in human gliomas (Fig. 2A). Additionally, the methylation analysis results revealed that most of the genes showed reduced levels of methylation (Fig. 2C). Of note, TP53 (46\%), which inhibits reprogramming, PGC-EGC conversion and oocyte development $[20,34,43,44]$; PTEN (7\%), which strongly inhibits PGC-EGC conversion [9, 34]; and NOTCH1 (8\%), which promotes pluripotency [34], were frequently detected among the genetic changes (Fig. 2A). TP53 and PTEN mutations were correlated with poor outcomes, while NOTCH1 mutations were associated with improved outcomes (Fig. 2B). The data indicated that epigenetic changes but not genetic changes, occurred in most of the core genes related to the embryonic/germ cell developmental axis in human gliomas, while the suppressor genes associated with reprogramming or PGC-EGC conversion frequently lost in gliomas with poor outcomes. Moreover, the reduced levels of methylation in the gene groups supported the activation of genes in gliomas.

\section{Links of the embryonic/germ cell development-like axis to a malignant prognosis}

Gliomas are pathologically classified as glioblastomas (GBM, grade WHO IV) and lower-grade gliomas (LGG, grades WHO II and WHO III) and have distinct malignant stages and outcomes [45]. We analysed the TCGA and GTEx dataset to compare the expression of serial genes related with embryonic/germ cell development between gliomas and normal brain tissues. Compared to normal brain tissues, the mRNA level of SOX2, MYC, NOTCH1, STAT3, BMP2, ACVR1, ITGB1, WNT5A, CXCR4 and ZP3 increased significantly in both GBM and LGG as well as the mRNA levels of LIF, PRDM1, IFITM3 and GDF15 increased significantly in GBM but not in LGG (Fig. S1). However, there was no significant differentiation in the mRNA levels of BMP4, BMP8B, SOX17, KIT, NANOS3, DND1, DAZL, DDX4, ROR2, SYCP3 and DMC1 between gliomas and normal brain tissues (not shown). To further determine whether the gene groups were activated in gliomas and linked to pathologic classification, malignant prognosis and clinical outcomes, we analysed the mRNA sequencing results of gliomas from the CGGA database. The data showed that IPS reprogramming factors and pluripotency-related genes (POU5F1, SOX2, KLF4, MYC and 
NOTCH1) were detected in almost all histologic types and grades of gliomas (Fig. 3A, B, and Fig. S2A, S3A, Table S3-S5). While the mRNA levels of genes related to pluripotency (SOX2, KLF4, MYC and NOTCH1) were not obviously correlated with the clinical glioma grade or outcome, the mRNA level of POU5F1, which also plays a crucial role in PGC specification, was often linked to a higher glioma grade and poor outcome (Fig. 3A, 3B, 4A, 4C, 4D and Fig. S4A, Table S3-S5). These findings revealed that the activation of reprogramming-related genes was commonly seen even in low-grade gliomas; however, it might be insufficient to lead to a malignant prognosis.

As expected, the mRNA sequencing results of gliomas from the CGGA database showed that the mRNA levels of various genes related to germ cell development were also enriched in some gliomas, including LIF, PRDM1, BMP2, BMP4, BMP8, ACVR1, IFITM3, ITGB1, CXCR4, WNT5A, ROR2, ZP3, GDF15, SOX17, DAZL, DDX4, SYCP3 and DMC1 (Fig. 3A, and Fig. S2, S3B, S3C, Table S3, S4). The mRNA levels of PRDM1, a core germ cell specification gene, as well as its core upstream gene ACVR1 and downstream gene IFITM3 were totally associated with advanced pathologic grades and poor outcomes (Fig. 3B, 4B-D, and Fig. S3B, S4A, Table S3-S5). High mRNA levels of genes related to PGC proliferation/survival/migration (ITGB1, CXCR4, WNT5A and ROR2), ES-PGC conversion (BMP8B and LIF), PGC-EGC conversion (LIF and STAT3), PGC survival (DAZL) and oocytes/early embryos (GDF15) also showed totally correlations with an advanced pathologic grade and poor overall survival of patients (Fig. 3B, 4B-D, and Fig. S3B, S4A, Table S3-S5). Notably, POU5F1 partners with SOX2 in human ES cells and partners with SOX17 in human PGCs [33, 36]. Although the mRNA levels of SOX17, a core germ cell specification gene [36], were enriched in some gliomas, they did not

show obvious correlations with advanced pathologic grades and poor outcomes (Fig. 3B, 3D, 4C, 4D and Fig. S3B, S4A, Table S3-S5), likely because SOX17 also inhibits PGC-EGC reprogramming [33]. The mRNA level of gene related with later meiosis (DMC1) was slightly associated with poor overall survival (Fig. 4C, 4D and Fig. S3B, S4A, Table S5). The mRNA levels of genes related to the activation of ES-PGC conversion but inhibition of PGC-EGC conversion (BMP2 and BMP4), late PGCs (DDX4), meiosis entry (SYCP3) as well as genes related to PGC fate determination (KIT, NANOS3 and DND1) showed no or negative correlations with advanced pathologic grades and poor outcomes (Fig. 3B, 4C and Fig. S3B, S4A, Table S3-S5). These data suggest that tumour cells arrested in the germ cell-like developmental stage (not early PGC-like stage) and that lost the ability to return to the embryonic cell-like state might not lead to a malignant prognosis of gliomas. Compared with LGG, GBM showed higher expression of POU5F1, PRDM1, BMP8B, LIF, STAT3, ACVR1, IFITM3, ITGB1, CXCR4, WNT5A, ROR2 and GDF15 (Fig. $3 \mathrm{~A}, 3 \mathrm{~B}$, and Fig S2A, Table S4), indicating that both the activation of the PGC-like state and the return of germ cells to the embryo-like state were essential in the malignant behaviours of some gliomas via somatic parthenogenetic embryo-like cycle and/or somatic PGC-EGC/ES-like cycle. Compared with LGG, secondary GBM arising from LGG [46] showed higher expression of PRDM1, BMP8B, ACVR1, LIF, STAT3, ITGB1, WNT5A, CXCR4, MYC but decreased expression of genes related to germ cell fate, including Nanos3, DND1, BMP2, BMP4, SOX17, DDX4, SYCP3, DMC1 and ZP3 (Fig. 3A, 3C, and Fig. S2A, Table S4), indicating that the PGC-EGC/ES-like conversion pathway rather than the mature development pathway might be activated in secondary GBM and that the activation of PGC-EGC/ES- 
like cycle was linked to the malignant prognosis of LGG. Compared with primary GBM, secondary GBM showed higher expression of ITGB1, ACVR1, MYC, STAT3 and BMP2 but significantly decreased expression of genes related to germ cell mature (Nanos3, DND1, DDX4, SOX17, SYCP3, DMC1, ZP3 and GDF15) and genes related to inhibition of PGC-EGC conversion (BMP4 and SOX17) (Fig. 3A, 3D, and Fig. S2A, Table S3), further indicating that the activation of PGC-EGC/ES-like conversion was one of driving events to malignant prognosis of gliomas. In addition, the decreased expression of genes related to germ cell development was accompanied by the decreased expression of GDF15, consistent with the fact that activation of gene GDF15 links to the oocyte/early embryo-like state (Fig. 3A, 3D, and Table

S3). Collectively, these findings indicated that the genes related to the activation of the parthenogenetic embryo-like cycle and activation of the PGC-EGC-like cycle were both linked to poor patient outcomes, which might represent two pathways that drive the malignant prognosis of gliomas.

\section{Inhibition of embryonic/germ cell cycle-related gene expression among gliomas with $1 \mathrm{p} 19 \mathrm{q}$ codeletion}

LGG have wide survival ranges, from 1 to 15 years, and distinct therapeutic sensitivities [45]. LGG with deletion of chromosome arms $1 p$ and 19q (1p19q codeletion) are often associated with impressive therapeutic sensitivities and favourable clinical outcomes [45]. Consequently, we determined whether genes related to the embryonic/germ cell developmental axis were inhibited in glioma samples with $1 p 19 q$ codeletion. The combined mRNA sequencing data from the 325 and 694 datasets of CGGA showed that the mRNA levels of POU5F1, LIF, BMP8B, ROR2, CXCR4, IFITM3, ITGB1 and GDF15 were extremely low in almost all glioma samples with 1p19q codeletion (1p19q-codel) compared to glioma samples without 1p19q codeletion (1p19q-noncodel) (Fig. 5A-C, and Table S6). Overall, the mRNA levels of ACVR1, STAT3, WNT5A, KLF4 and DMC1 were obviously decreased in glioma samples with $1 p / 19 q$ codeletion compared to those in glioma samples without $1 p 19 q$ codeletion (Fig. 5A-C, and Fig. S5, Table S6). However, 1p19q codeletion did not inhibit the mRNA levels of SOX2, SOX17, SYCP3, MYC, ZP3, NOTCH1, DDX4, KIT, NANOS3, DND1, BMP2 and BMP4 (Fig. 5A-C, and Fig. S5, Table S6). These findings indicated that $1 \mathrm{p} 19 \mathrm{q}$ codeletion robustly inhibited genes involved in promoting the embryonic/germ cell cycle.

\section{Clinical significance of embryonic/germ cell cycle-related molecular groups among gliomas}

Histopathological classification is often performed in gliomas; however, this method is not sufficient to predict clinical outcomes [45]. Consequently, we performed an analysis of the molecular group related to the embryonic/germ cell cycle (including LIF, STAT3, PRDM1, IFITM3, ACVR1, CXCR4, WNT5A, ROR2, ITGB1, POU5F1, GDF15 and BMP8B) (Table S7) to determine whether we could identify glioma outcomes more accurately based on the germ cell-related molecular group than based on the histologic class. The combined mRNA sequencing data of 325 and 694 CGGA datasets showed that 
patients with higher expression of genes in the molecular group had much poorer overall survival than patients with lower expression of genes in the gene groups (Fig. 6A). The median survival of patients in the two groups was 443 days (high) and 3,411 days (low) respectively (Fig. 6A, and Table S8). Notably, increased expression of genes in the molecular group was frequently observed in the same gliomas (Fig. 6B). Approximately $71.92 \%$ of gliomas with increased expression of genes in the molecular group had at least two genes with increased expression (Fig. 6B). Among grade II-primary, II-recurrence, IIIprimary, II-recurrence, IV-primary, IV-recurrence gliomas and IV-secondary gliomas, the total ratio of gene groups with high expression of a single gene was $24.71 \%, \sim 52.50 \%, \sim 40.88 \%, \sim 58.3 \%, \sim 83.98 \%$, $\sim 89.91 \%$ and $\sim 100 \%$, respectively (Fig. $6 \mathrm{C}$, and Table S9), further indicating that activation of the embryonic/germ cell-like developmental axis may be associated with the pathological grade, recurrence and progression of gliomas. Interestingly, the mRNA profiles of the molecular group could be used to separate poor outcomes from improved outcomes among patients who harboured gliomas with the same WHO grade, especially among the patients with grade III gliomas (Fig. 6C, and Table S8, S10). Median survival was 344 days (higher) and 710 days (lower) among patients with grade IV gliomas, 544 days (higher) and 2,633 days (lower) among patients with grade III gliomas, and 2,219 days (higher) and more than 5,000 days (lower) among patients with grade II gliomas (Fig. 6D, and Table S8). Notably, glioma classification based on the WHO grade and molecular group could predict clinical behaviours more accurately (Fig. 6E, Fig. S6A and Table S8, S10). Subtype grade II gliomas with lower expression of genes in the molecular group had favourable clinical outcomes (Fig. 6E and Table S8). Grade III gliomas with higher expression of genes in the molecular group were similar to grade IV gliomas in terms of clinical outcomes (Fig. 6E, and Table S8).

Among patients who had gliomas with 1p19q codeletion in the combined dataset, patients with higher expression of genes in the gene group (median survival=1265 days) had much poorer overall survival than patients with lower expression of genes in the molecular group (median survival> 5000 days) (Fig. $6 \mathrm{~F}$ and Table S8). Among the gliomas without $1 \mathrm{p} 19 \mathrm{q}$ codeletion in the combined dataset, the median survival of the two groups of patients was 415 days (higher) and 2382 days (lower) (Fig. 6F and Table S8) respectively. Classification by combining the $1 p 19 q$ status and the molecular group could subdivide gliomas into four subtypes with distinct clinical outcomes (Fig. 6F, G and Table S8), suggesting a possible molecular method to predict clinical behaviour. These findings indicate that the embryonic/germ cell cycle-related molecular group can be used as a good marker for predicting clinical outcomes and may be associated to the low-glioma progression to advanced gliomas.

\section{Appearance of embryonic/germ cell-like cells in gliomas}

We then investigated whether embryonic/germ cell-like cells were present in human gliomas and linked to malignant traits. HE and immune staining showed that germ cell-like cells could be observed in some human glioma tissues, and the appearance of germ cell-like cells was associated with the pathological grade of gliomas (Fig. 7A, and Fig. S6). Compared with LGG, germ cell-like cells were easily observed in GBM (Table S11). Notably, a series of embryonic/germ cell-like cells at different developmental stages 
could be observed in the same tumour tissues among some grade IV gliomas (approximately 40\%), including PGC-like cells, oocyte-like cells and parthenogenetic preimplantation embryo-like cells, indicating that a somatic parthenogenetic embryo-like cycle might be present in some GBM (Fig. 7B, and Fig. S6). It was documented that polyploid giant cancer cells (PGCCs) were blastomere-like cells $[1,47]$. Taken together, it is possible that activation of the embryonic/germ cell-like developmental axis occurs during tumour initiation and malignant prognosis.

\section{Discussion}

Our findings showed that germ cell-like cells were present in cultured glioma cells and glioma tissues and that deletion of DAZL repressed both PGC-like cell formation and tumour initiation in human glioma cell lines. Moreover, the mRNA sequencing results showed that a series of genes related to reprogramming, PGC specification and germ cell development could be detected in most gliomas. Together with reports that embryonic/germ cell-specific genes are essential in malignant tumour behaviours [17-27, 48], it is possible that activation of the embryonic/germ cell-like state may be a necessary precondition for glioma development, which can explain why gliomas show high embryonic/germ cell traits and extensively express genes related to embryonic/germ cell development and testis antigens of tumours $[2,5,15,16$, $24,26]$.

However, our findings indicated that reprogramming activation or germ cell-like state activation alone seemed to be insufficient to lead to a malignant prognosis. In contrast, the increased mRNA levels of genes related to the activation of the embryonic/germ cell-like cycle showed strong correlations with malignant prognoses and poor clinical outcomes, possibly because this cycle could lead to tumours with migratory PGC-like cells (which are linked to metastasis) and a naive ES/PGC-like state (which is linked to tumourigenicity) through the somatic parthenogenetic embryo-like cycle or somatic PGC-EGC-like cycle. Notably, it was shown that some core oncogenes/tumour suppressors strongly upregulated/repressed IPS reprogramming (TP53, RB, MYC) [43], PGC-like cell formation (TP53), PGC-EGC conversion (TP53, PTEN) [9], meiosis (TP53) [49] and primary oocyte maturation (TP53) [20, 50], which further supported the essential role of the embryonic/germ cell-like cycle in malignant tumour prognosis [51]. In summary, our findings indicated that a crucial role of germ cell-like cell formation in glioma initiation as well as activation of the parthenogenetic embryo-like cycle and PGC-EGC-like cycle might represent two driving events in the malignant prognosis of gliomas, which may provide a novel way to better understand the nature of glioma and develop targeted therapies for gliomas as well as important markers for predicting clinical outcomes in gliomas.

\section{Abbreviations}

CGGA: Chinese Glioblastoma Genome Atlas; EC cells: embryonal carcinoma cells; EC cells: embryonal carcinoma cells; EGCs: embryonic germ cells; ES cells: embryonic stem cells; iPS cells: induced pluripotent stem cells; PGCs: primordial germ cells; GTEx: the Genotype-Tissue Expression; TCGA: the Cancer Genome Atlas 


\section{Declarations}

\section{Acknowledgements}

The genetic analysis and clinical outcome results are based on data generated from the Chinese Glioma Genome Atlas (CGGA) (http://cgga.org.cn/index.jsp), the Cancer Genome Atlas (TCGA) and the GenotypeTissue Expression (GTEx). Some data were analysed using the http://gepia2.cancer-pku.cn/\#analysis.

\section{Author contributions:}

Z. M., and Z.F. performed the experiments and analysed the data from the Chinese Glioma Genome Atlas (CGGA). H. Z. provided the glioma tissues and pathologic information. HK. L provided some advice. C.L. designed the experiments, analysed the data and wrote the paper.

\section{Funding}

This work was supported by the Natural Science Foundation of China (No. 81372351), the Natural Science Foundation of Shanghai (21ZR1410900) and Shanghai Municipal Key Clinical Specialty of China (shslczdzk03303).

\section{Availability of supporting data}

All data are available in the main text or in the supplementary materials.

\section{Consent for publication}

Not applicable

\section{Conflict of interest}

The authors declare that they have no conflicts of interest.

\section{References}

1. Niu, N., Mercado-Uribe, I. \& Liu, J. Dedifferentiation into blastomere-like cancer stem cells via formation of polyploid giant cancer cells., 36, 4887-4900 (2017).

2. Simpson, A. J., Caballero, O. L., Jungbluth, A., Chen, Y. T. \& Old, L. J. Cancer/testis antigens, gametogenesis and cancer. Nat Rev Cancer, 5, 615-625 (2005). 
3. Brewer, B. G., Mitchell, R. A., Harandi, A. \& Eaton, J. W. Embryonic vaccines against cancer: an early history. Exp Mol Pathol, 86, 192-197 (2009).

4. Bignold, L. P., Coghlan, B. L. \& Jersmann, H. P. Hansemann, Boveri, chromosomes and the gametogenesis-related theories of tumours. Cell Biol Int, 30, 640-644 (2006).

5. Old, L. J. Cancer/testis (CT) antigens - a new link between gametogenesis and cancer. Cancer Immun, 1, 1 (2001).

6. Stevens, L. C. The development of transplantable teratocarcinomas from intratesticular grafts of preand postimplantation mouse embryos. Dev Biol, 21, 364-382 (1970).

7. Stevens, L. C. Origin of testicular teratomas from primordial germ cells in mice. J Natl Cancer Inst, 38, 549-552 (1967).

8. Stevens, L. C. \& Little, C. C. Spontaneous Testicular Teratomas in an Inbred Strain of Mice. Proc Nat/ Acad Sci U S A, 40, 1080-1087 (1954).

9. Kimura, T. et al. Conditional loss of PTEN leads to testicular teratoma and enhances embryonic germ cell production. Development, 130, 1691-1700 (2003).

10. Thomson, J. A. et al. Embryonic stem cell lines derived from human blastocysts., 282, 1145-1147 (1998).

11. Takahashi, K. et al. Induction of pluripotent stem cells from adult human fibroblasts by defined factors., 131, 861-872 (2007).

12. Ben-Porath, I. et al. An embryonic stem cell-like gene expression signature in poorly differentiated aggressive human tumors. Nat Genet, 40, 499-507 (2008).

13. Ezeh, U. I., Turek, P. J., Reijo, R. A. \& Clark, A. T. Human embryonic stem cell genes OCT4, NANOG, STELLAR, and GDF3 are expressed in both seminoma and breast carcinoma., 104, 2255-2265 (2005).

14. Rudin, C. M. et al. Comprehensive genomic analysis identifies SOX2 as a frequently amplified gene in small-cell lung cancer. Nat Genet, 44, 1111-1116 (2012).

15. Guo, Y. et al. Expression profile of embryonic stem cell-associated genes Oct4, Sox2 and Nanog in human gliomas., 59, 763-775 (2011).

16. Son, M. J., Woolard, K., Nam, D. H., Lee, J. \& Fine, H. A. SSEA-1 is an enrichment marker for tumorinitiating cells in human glioblastoma., 4, 440-452 (2009).

17. Mu, P. et al. SOX2 promotes lineage plasticity and antiandrogen resistance in TP53- and RB1deficient prostate cancer., 355, 84-88 (2017).

18. Boumahdi, S. et al. SOX2 controls tumour initiation and cancer stem-cell functions in squamous-cell carcinoma. Nature, 511, 246-250 (2014).

19. Liu, C. et al. Germline traits of human hepatoblastoma cells associated with growth and metastasis. Biochem Biophys Res Commun, 437, 120-126 (2013).

20. Liu, C. et al. Abnormal gametogenesis induced by $\mathrm{p} 53$ deficiency promotes tumor progression and drug resistance. Cell Discov, 4, 54 (2018). 
21. Liu, C. et al. Identification of primordial germ cell-like cells as liver metastasis initiating cells in mouse tumour models. Cell Discov, 6, 15 (2020).

22. Moriya, C. et al. Inhibition of PRDM14 expression in pancreatic cancer suppresses cancer stem-like properties and liver metastasis in mice., 38, 638-648 (2017).

23. Chen, C. L. et al. NANOG Metabolically Reprograms Tumor-Initiating Stem-like Cells through Tumorigenic Changes in Oxidative Phosphorylation and Fatty Acid Metabolism. Cell Metab, 23, 206219 (2016).

24. Zhang, F. et al. Suppressing Dazl modulates tumorigenicity and stemness in human glioblastoma cells. BMC Cancer, 20, 673 (2020).

25. Adhikari, A. S. et al. CD117 and Stro-1 identify osteosarcoma tumor-initiating cells associated with metastasis and drug resistance. Cancer Res, 70, 4602-4612 (2010).

26. Janic, A., Mendizabal, L., Llamazares, S., Rossell, D. \& Gonzalez, C. Ectopic expression of germline genes drives malignant brain tumor growth in Drosophila., 330, 1824-1827 (2010).

27. Kaufman, C. K. et al. A zebrafish melanoma model reveals emergence of neural crest identity during melanoma initiation., 351, aad2197 (2016).

28. Liu, C. et al. Activation of the germ-cell potential of human bone marrow-derived cells by a chemical carcinogen. Sci Rep, 4, 5564 (2014).

29. Liu, C. et al. Generation of pluripotent cancer-initiating cells from transformed bone marrow-derived cells. Cancer Lett, 303, 140-149 (2011).

30. Ma, Z. et al. Spontaneous generation of germline characteristics in mouse fibrosarcoma cells. Sci Rep, 2, 743 (2012).

31. Ma, Z. et al. Spontaneous germline potential of human hepatic cell line in vitro. Mol Hum Reprod, 19, 216-226 (2013).

32. Donehower, L. A. et al. Mice deficient for $\mathrm{p} 53$ are developmentally normal but susceptible to spontaneous tumours. Nature, 356, 215-221 (1992).

33. Oosterhuis, J. W. \& Looijenga, L. H. J. Human germ cell tumours from a developmental perspective. Nat Rev Cancer, 19, 522-537 (2019).

34. Saitou, M. \& Yamaji, M. Primordial germ cells in mice. Cold Spring Harb Perspect Bio/2012, 4.

35. Strome, S. \& Updike, D. Specifying and protecting germ cell fate. Nat Rev Mol Cell Biol, 16, 406-416 (2015).

36. Tang, W. W., Kobayashi, T., Irie, N., Dietmann, S. \& Surani, M. A. Specification and epigenetic programming of the human germ line. Nat Rev Genet, 17, 585-600 (2016).

37. Fukunaga, N. et al. Leukemia inhibitory factor (LIF) enhances germ cell differentiation from primate embryonic stem cells. Cell Reprogram, 12, 369-376 (2010).

38. Tarone, G. et al. Expression of beta 1 integrin complexes on the surface of unfertilized mouse oocyte. Development, 117, 1369-1375 (1993). 
39. Anderson, R. et al. Mouse primordial germ cells lacking beta 1 integrins enter the germline but fail to migrate normally to the gonads. Development, 126, 1655-1664 (1999).

40. Makoolati, Z., Movahedin, M. \& Forouzandeh-Moghadam, M. In vitro germ cell differentiation from embryonic stem cells of mice: induction control by BMP4 signalling.Biosci Rep2016, 36.

41. Zdravkovic, T. et al. Human stem cells from single blastomeres reveal pathways of embryonic or trophoblast fate specification. Development, 142, 4010-4025 (2015).

42. Soucek, K. et al. Presence of growth/differentiation factor-15 cytokine in human follicular fluid, granulosa cells, and oocytes. J Assist Reprod Genet, 35, 1407-1417 (2018).

43. Kawamura, T. et al. Izpisua Belmonte JC: Linking the p53 tumour suppressor pathway to somatic cell reprogramming. Nature, 460, 1140-1144 (2009).

44. Yamada, Y., Davis, K. D. \& Coffman, C. R. Programmed cell death of primordial germ cells in Drosophila is regulated by p53 and the Outsiders monocarboxylate transporter. Development, 135, 207-216 (2008).

45. Cancer Genome Atlas et al. Comprehensive, Integrative Genomic Analysis of Diffuse Lower-Grade Gliomas. N Engl J Med, 372, 2481-2498 (2015).

46. Ohgaki, H. \& Kleihues, P. The definition of primary and secondary glioblastoma. Clin Cancer Res, 19, 764-772 (2013).

47. Liu, J. The "life code": A theory that unifies the human life cycle and the origin of human tumors. Semin Cancer Biol, 60, 380-397 (2020).

48. Liu, X. et al. IFITM3 promotes bone metastasis of prostate cancer cells by mediating activation of the TGF-beta signaling pathway. Cell Death Dis, 10, 517 (2019).

49. Habu, T. et al. p53 Protein interacts specifically with the meiosis-specific mammalian RecA-like protein DMC1 in meiosis., 25, 889-893 (2004).

50. Bolcun-Filas, E., Rinaldi, V. D., White, M. E. \& Schimenti, J. C. Reversal of female infertility by Chk2 ablation reveals the oocyte DNA damage checkpoint pathway., 343, 533-536 (2014).

51. Liu, C., Moten, A., Ma, Z. \& Lin, H. K. The foundational framework of tumors: Gametogenesis, p53, and cancer. Seminars in cancer biology 2021. doi: 10.1016/j.semcancer.2021.04.018. Online ahead of print.

\section{Figures}



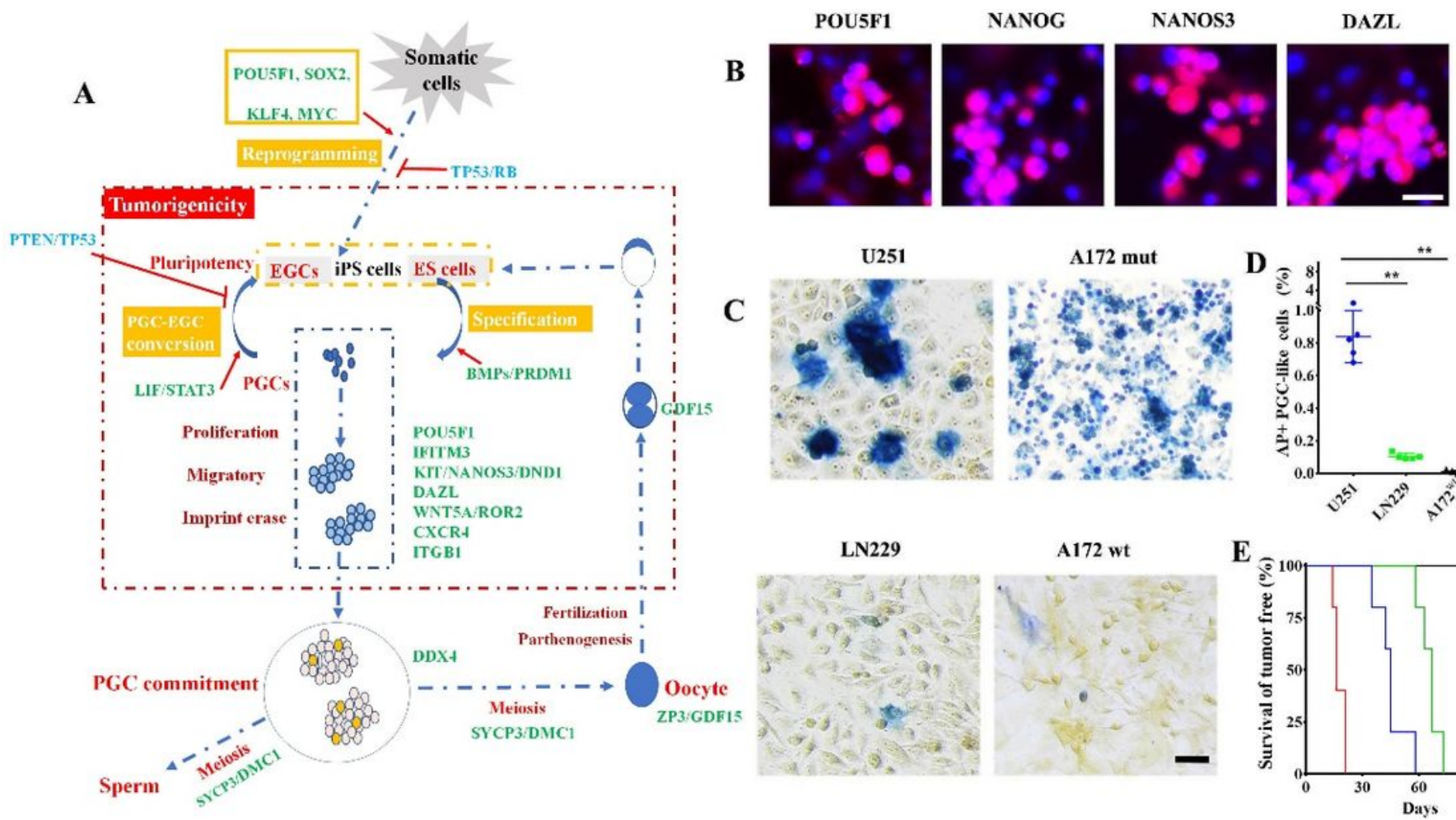

C
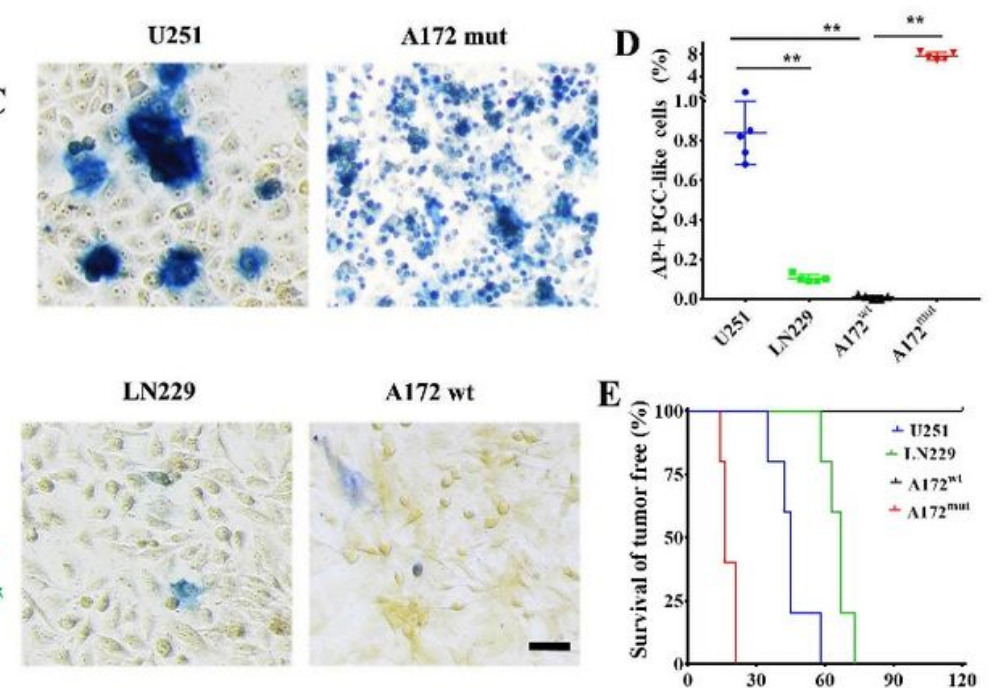

A172 wt
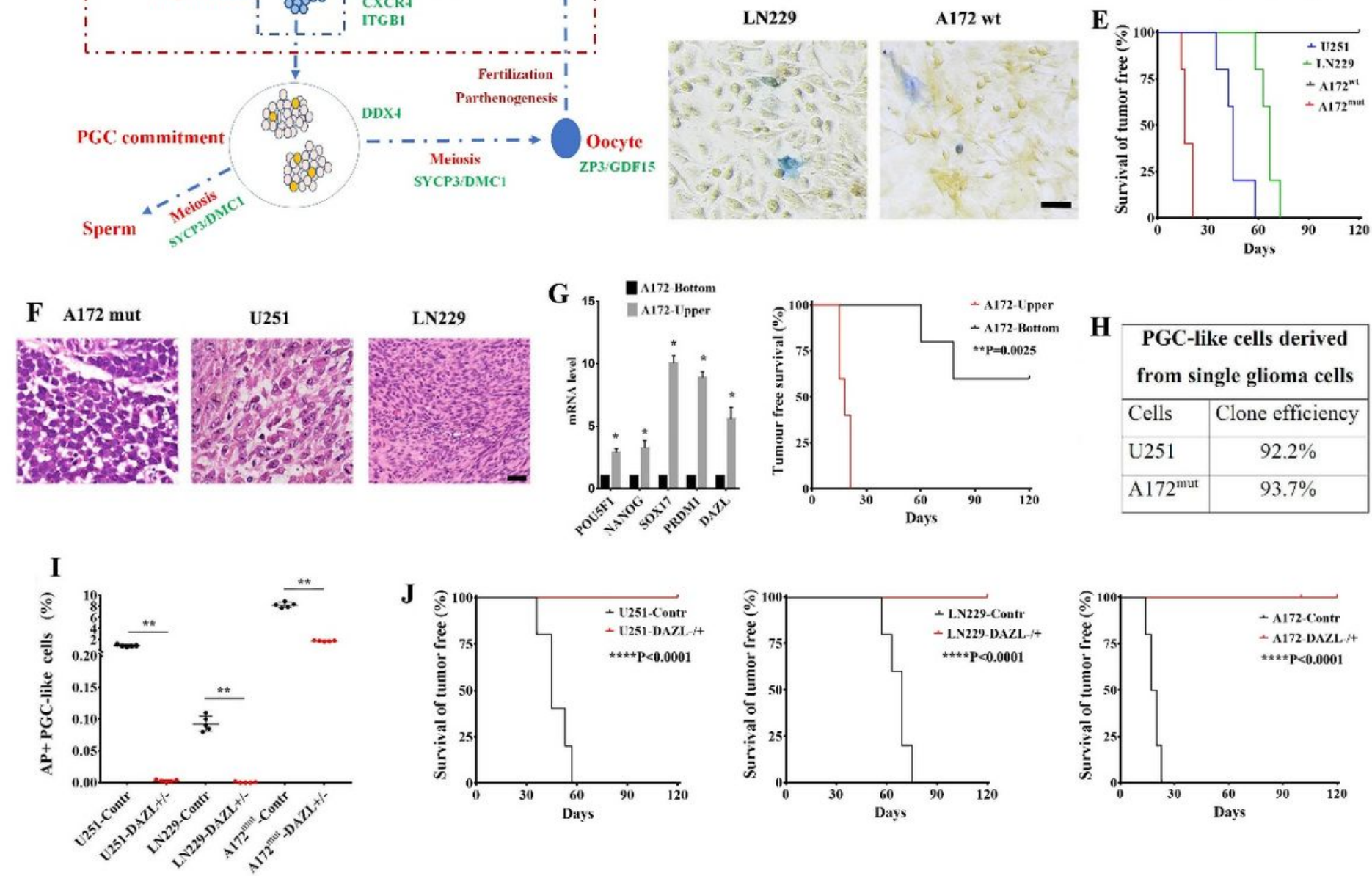

\section{Figure 1}

Experimental hypothesis and the crucial role of germ cell-like cell formation in tumour initiation. (A) Experimental hypothesis. (B) Immunofluorescence assays showed the expression and colocalization of the indicated antigens in cultured U251 cells. (C) AP staining showing PGC-like cells in U251, LN229, A172wt and A172mut glioma cells. (D) The percentage of AP-positive cells in glioma cultured cells. (E) Survival curve of tumour-free glioma cells showing their different tumour initiation abilities. (F) Image of tumour tissues (HE staining) from grafted mice. (G) The percentage of AP-positive cells in glioma 
cultured cells. (H) The efficiency of generating clones of PGC-like cells from single glioma cells. (I) The percentage of AP-positive cells in the control and DAZL+/- glioma cells. $(\mathrm{J})$ The Survival curve of tumourfree cells showing the tumour initiation ability of the control and DAZL+/- glioma cells. None of the DAZL+/- cell lines could cause tumours within 100 days after grafting in nude mice. Scale bar=20 $\mu \mathrm{m}$ in $(\mathrm{F}), 50 \mu \mathrm{m}$ in $(\mathrm{B}, \mathrm{C}) .{ }^{*} \mathrm{P}<0.01$.
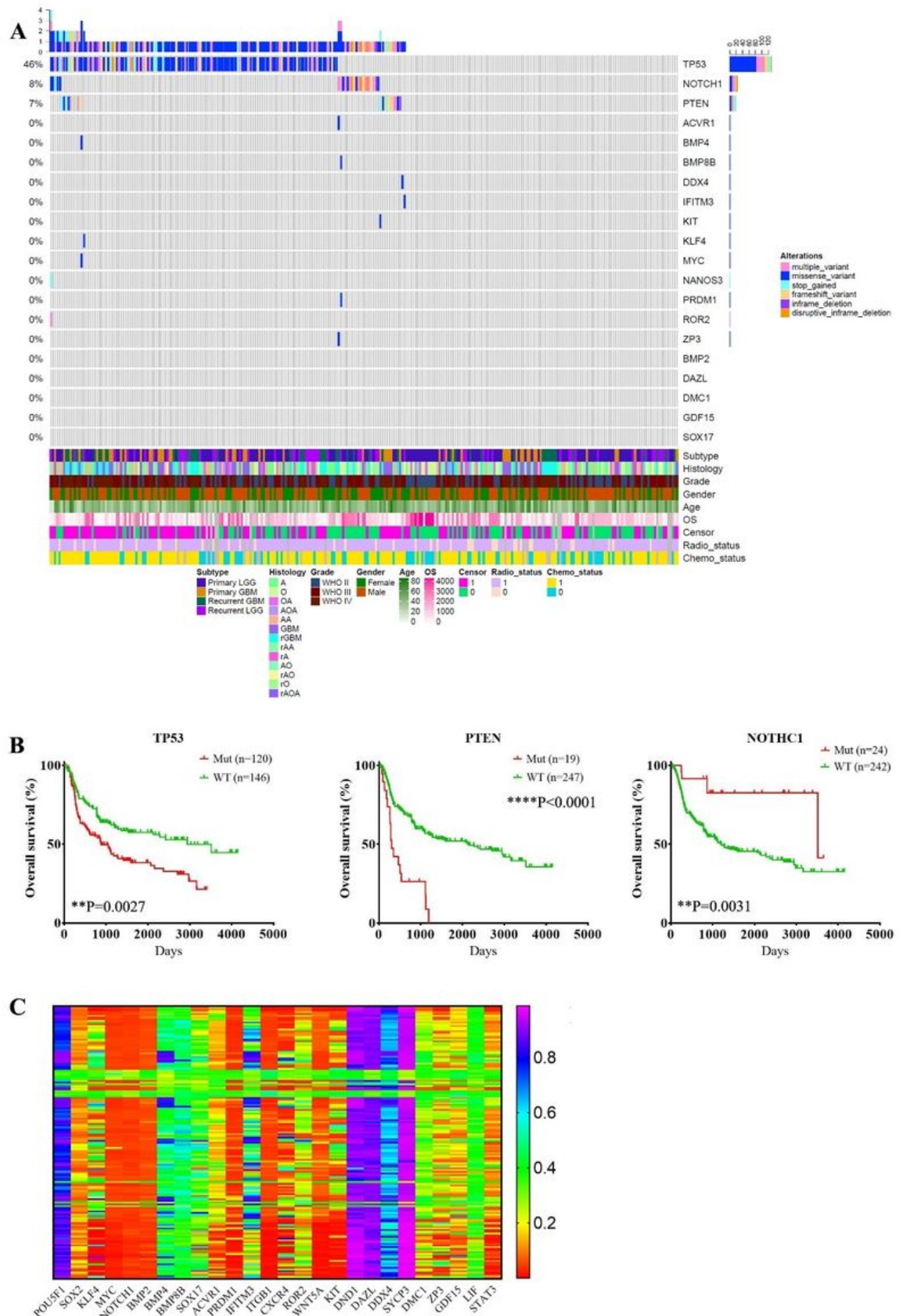

Figure 2 
Genetic and epigenetic changes in genes related to embryonic/germ cell development. (A) Whole-exome sequencing data showing the mutational landscape of various genes (the results of POU5F1, SOX2, STAT3, LIF, ITGB1, CXCR4, WNT5A, SYCP3 and DMC1 were not found in the CGGA database). (B) Overall survival (OS) curve showing the association of DNA changes in TP53, PTEN and NOTCH1 with the outcomes of gliomas. (C) Methylation data showing the methylation state of various genes.

A
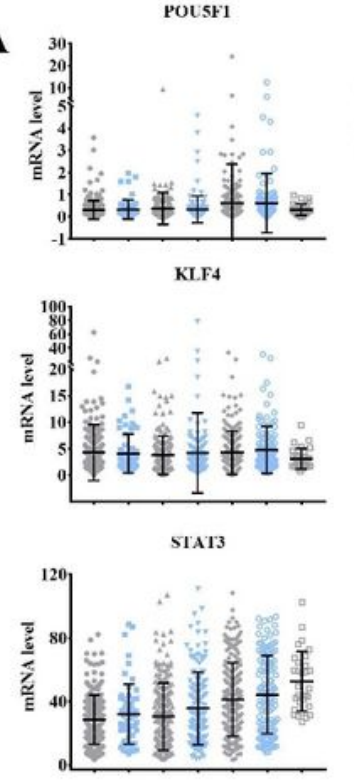

ACVR1
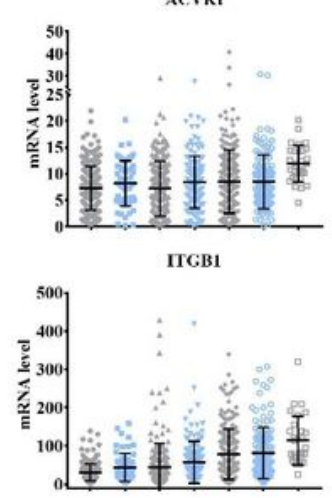

NANOS3
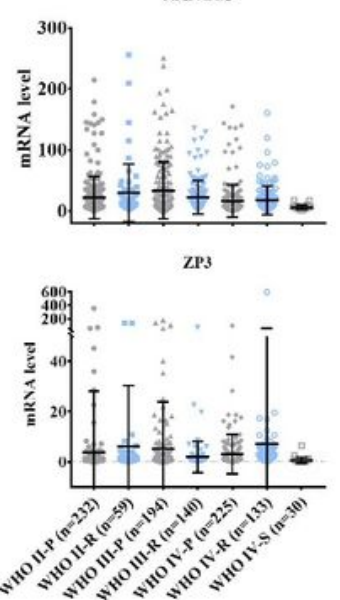
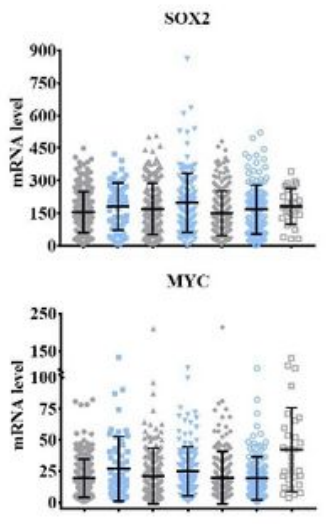

L.IF

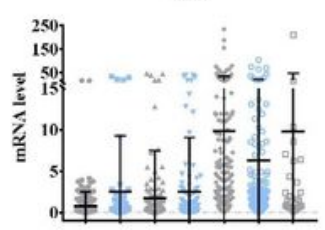

PRDM1
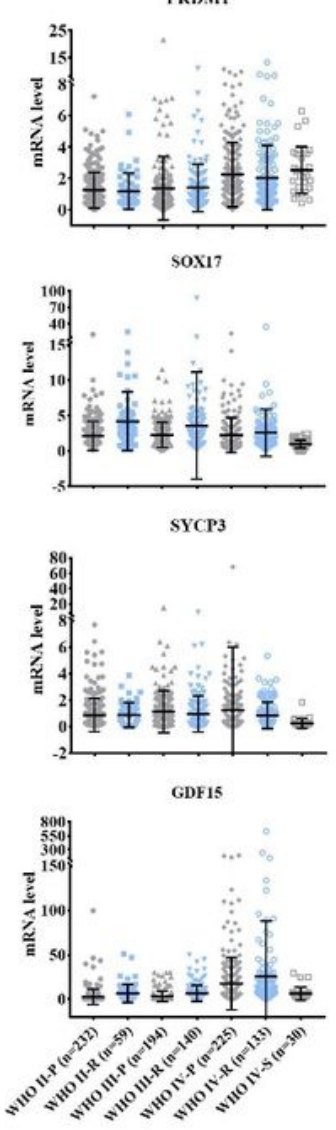

B
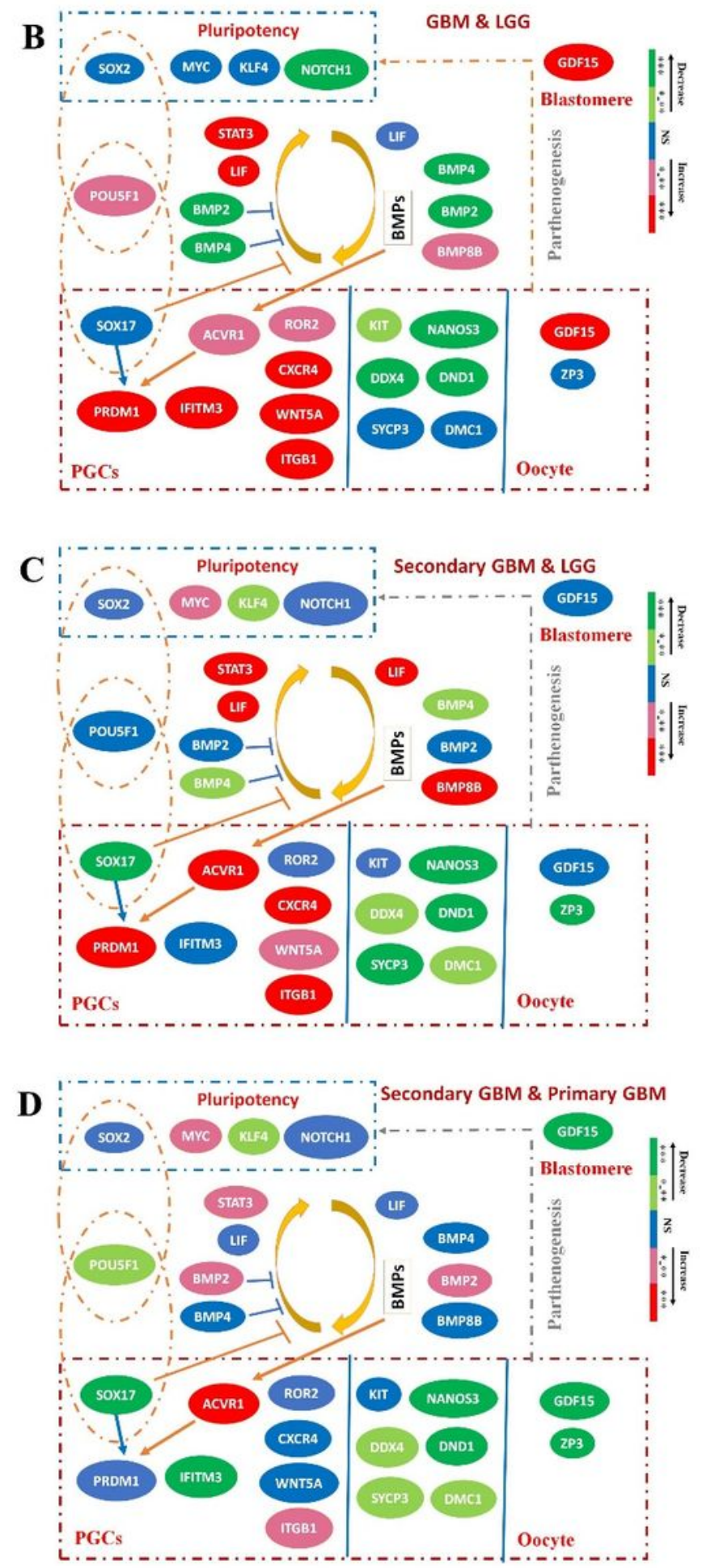

Figure 3 
Association between the expression of genes related to embryonic/germ cell development and the pathologic grades/subtypes. (A) RNA sequencing data showing the mRNA expression profile of various genes related to embryonic/germ cell development in subtypes of gliomas that had different pathologic grades. Differentiation of the mRNA levels of embryonic/germ cell-related genes in glioblastomas (GBM) versus lower-grade gliomas (LGG) (B), secondary GBM versus LGG (C). secondary GBM versus primary GBM (D). ${ }^{*} P<0.05,{ }^{*} P<0.01, * \star * P<0.001, * \star \star * P<0.0001$.
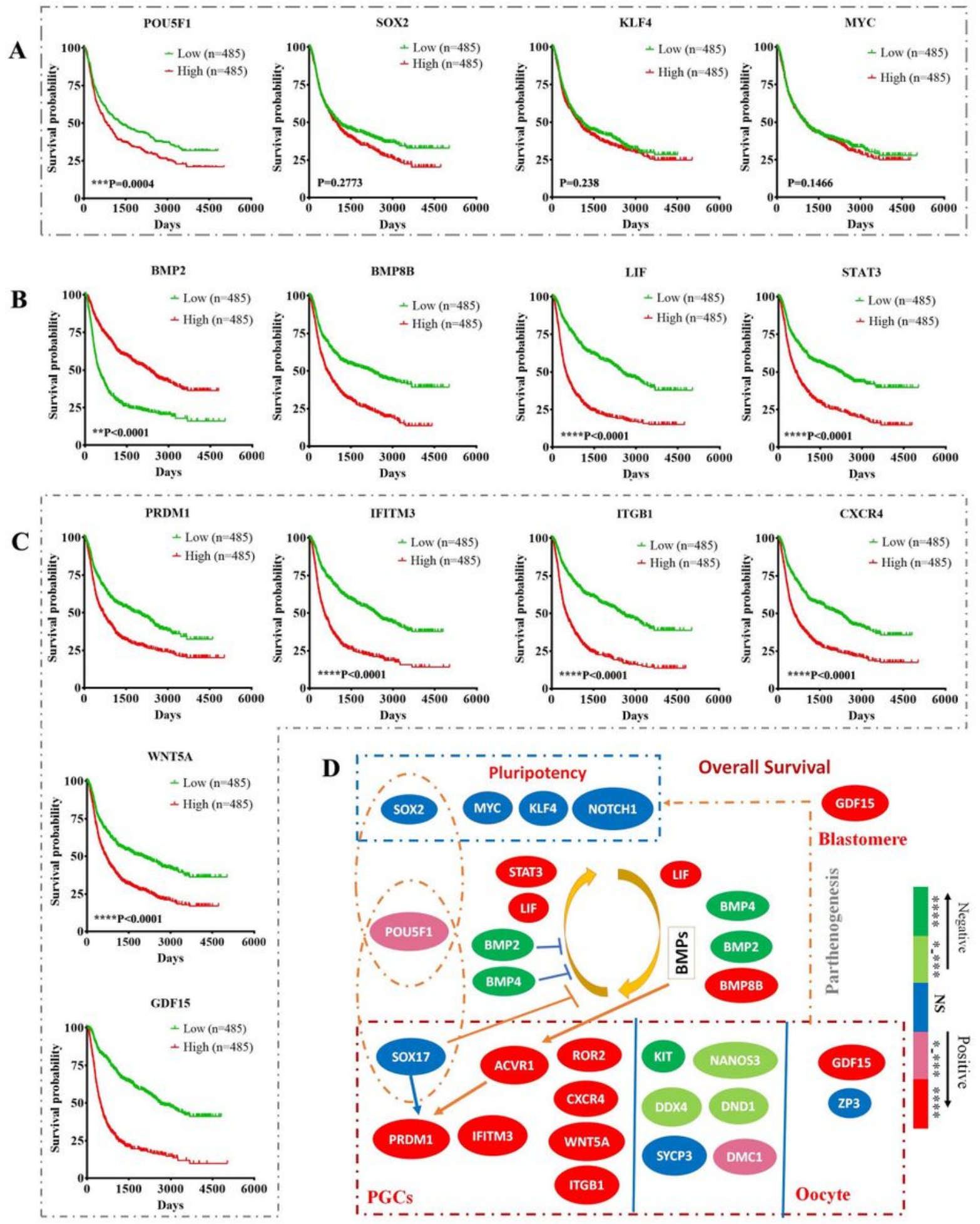
Association between the embryonic/germ cell related gene expression and outcomes of gliomas. RNA sequencing and clinical data showing the mRNA expression profiles of various genes related to IPS reprogramming (A) and germ cell development and early blastomere formation (B) in gliomas that had different outcomes. (C) Relationship between the mRNA level of embryonic/germ cell-related genes and overall survival of patients. (D) The role of the embryonic/germ cell related gene expression in outcomes of gliomas. ${ }^{*} \mathrm{P}<0.05,{ }^{*} \mathrm{P}<0.01,{ }^{*} * \mathrm{P}<0.001,{ }^{*} * * \mathrm{P}<0.0001$.

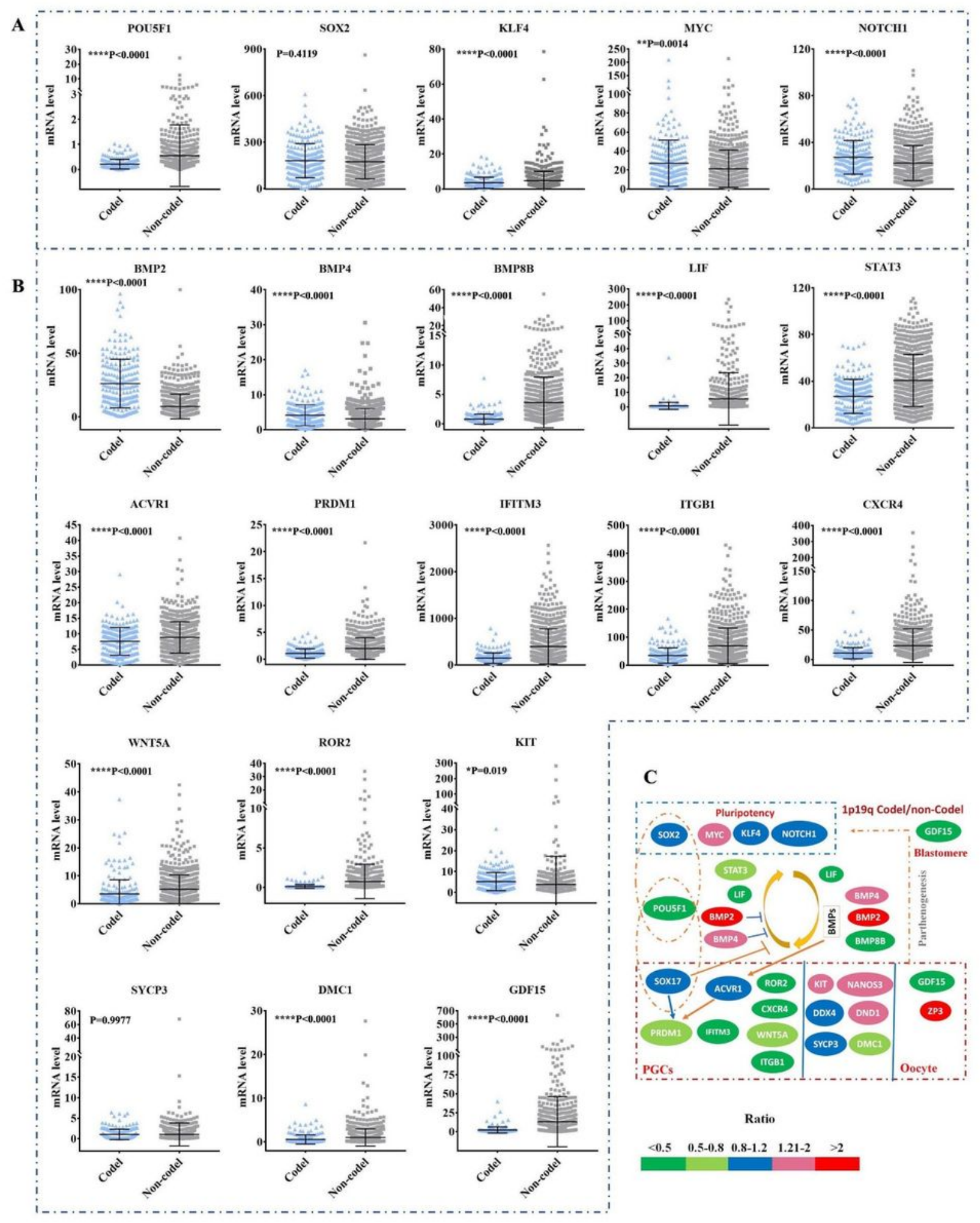


Association between the mRNA levels of various genes and 1p/19q codeletion status in gliomas. RNA sequencing and clinical data showing that the relationship between mRNA levels of genes related to IPS reprogramming and $1 p / 19 q$ codeletion status $(A)$; between $m R N A$ levels of genes related to germ cell development and early blastomere formation and $1 p / 19 q$ codeletion status (B). (C) The ratio of gliomas with $1 p / 19 q$ codeletion to gliomas without $1 p / 19 q$.
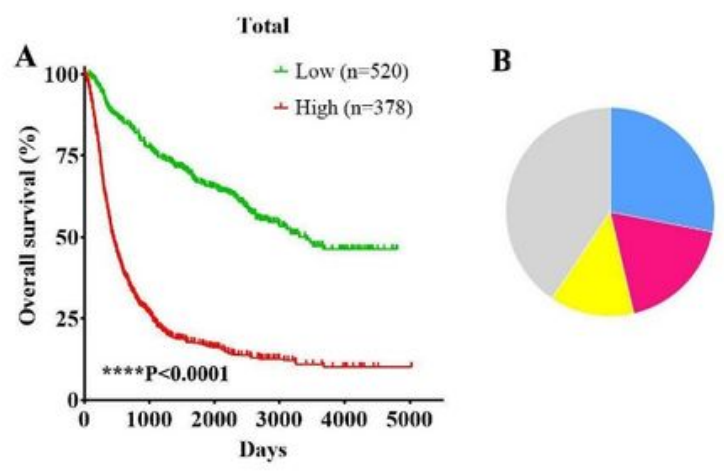

$\square 28.08 \%$ single-positive

$18.27 \%$ two-positive

$\square 13.08 \%$ three-positive

$\square 40.58 \%>$ three-positive

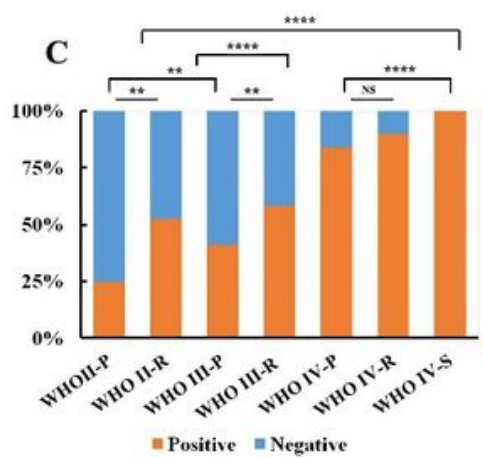

D

WHO II-single

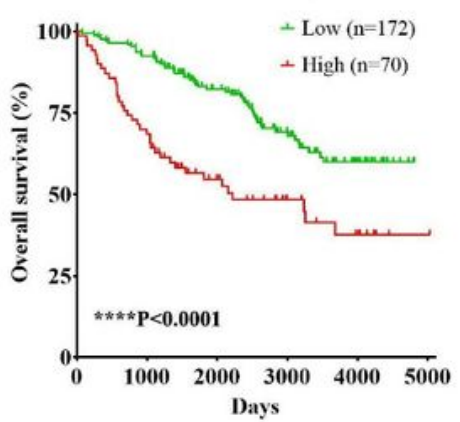

WHO III-single

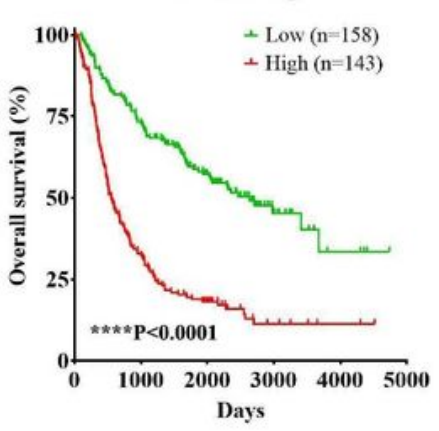

WIIO IV-single
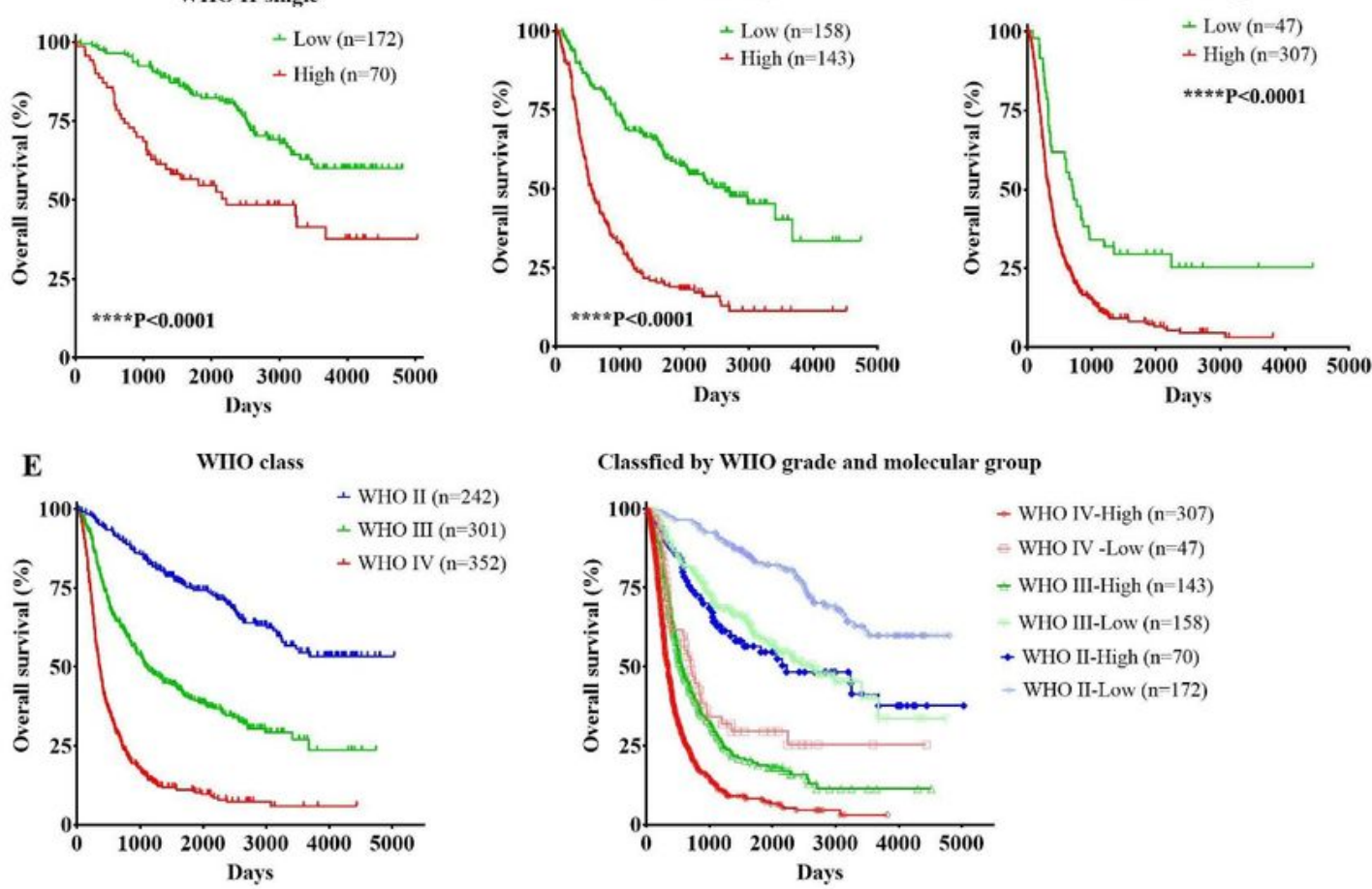

Classfied by WIIO grade and molecular group
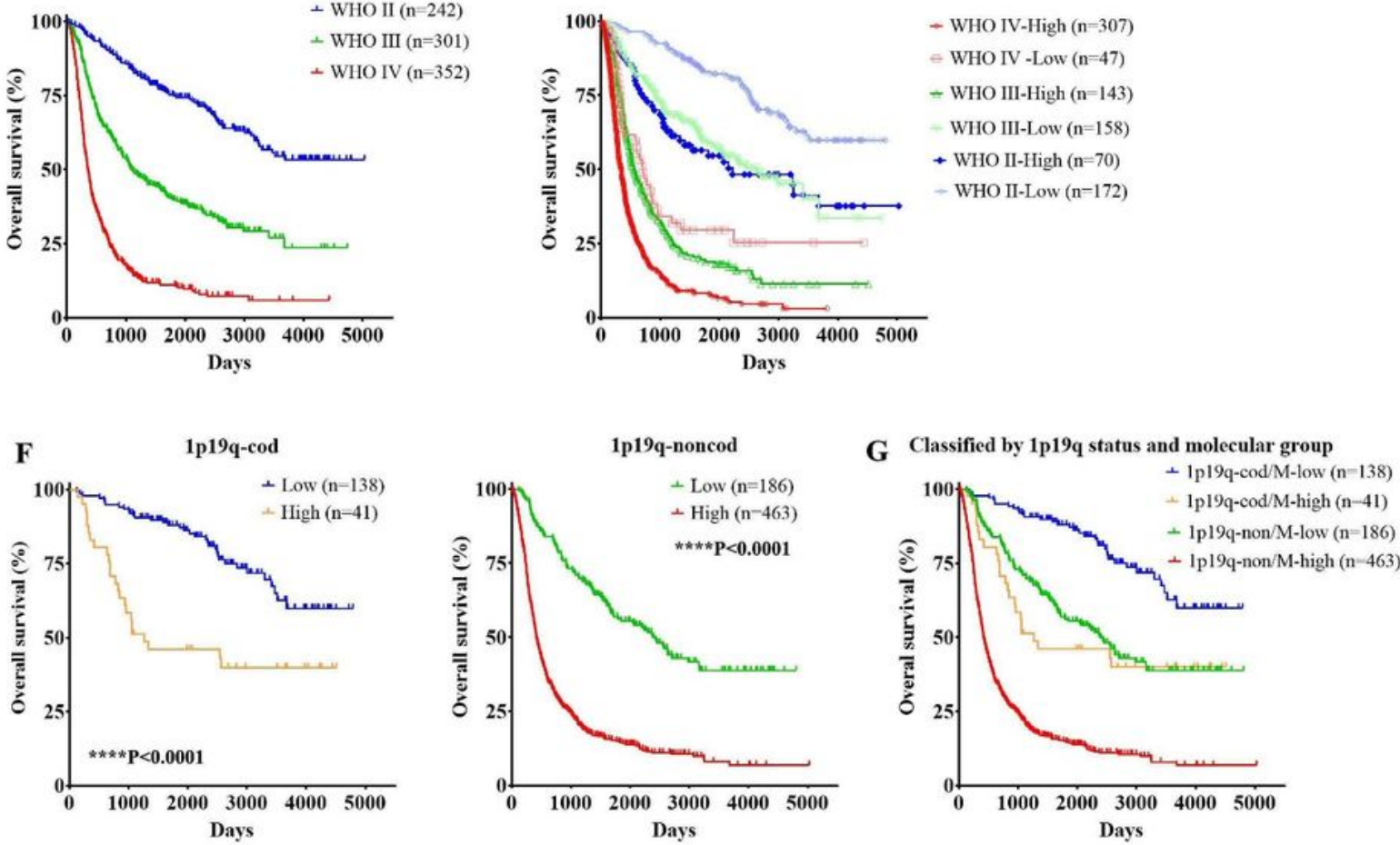

Figure 6 
Clinical significance and prevalence of molecular groups among gliomas. (A) Patients separated by the mRNA level of genes in the gene group showed different overall survival. (B) Distribution of gliomas with high expression of single to multiple genes in molecular groups among gliomas with higher expression of genes in molecular groups. (C) Prevalence of the molecular groups among distinct pathological grades. (D) Patients with the same pathological grade separated by the mRNA level of genes in the gene group showed different overall survival. (E) Gliomas classified according to the WHO grade or the WHO combined with the molecular group showed different overall survival. (F) Patients with the same 1p19q status classified by the molecular group showed different overall survival. (G) Gliomas classified according to 1p19q status and the molecular group showed different overall survival. 
HE

POU5F1

SOX2

NANOG
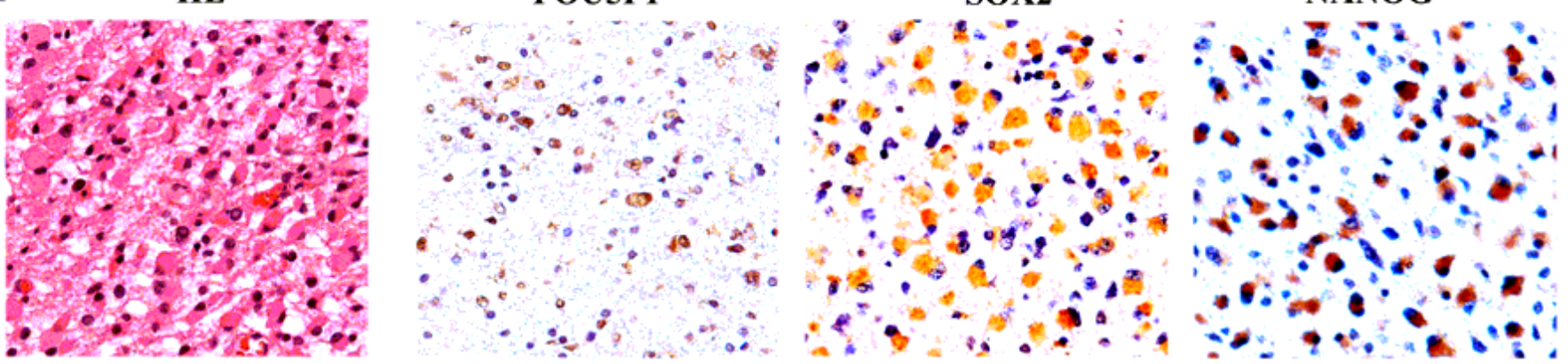

NANOS3

DAZL

DDX4
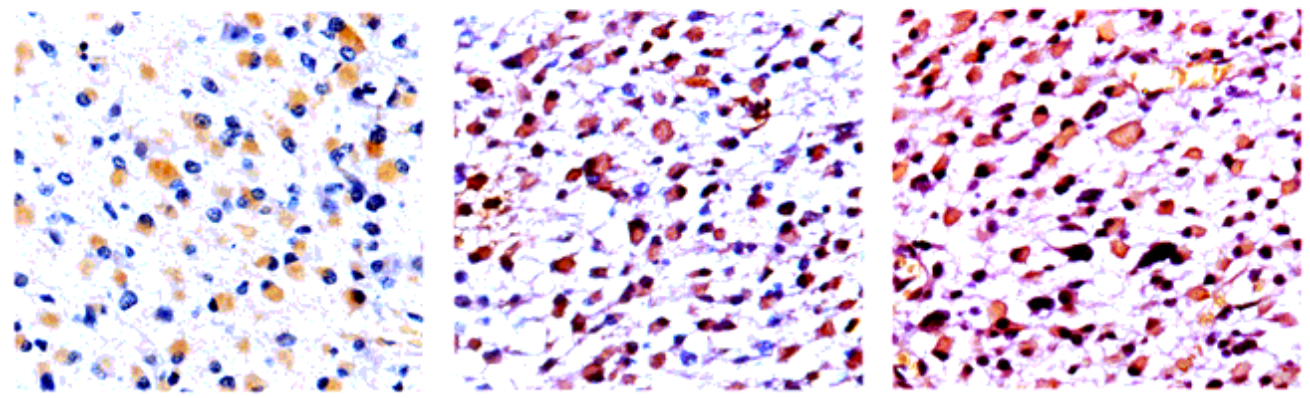

B

HE

POU5F1

SOX2

NANOG
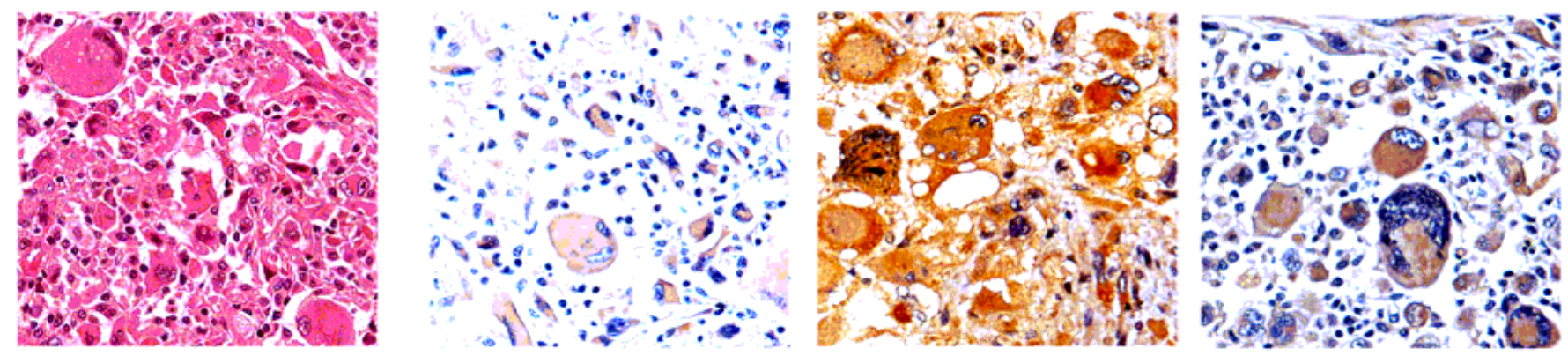

NANOS3

DAZL

DDX4
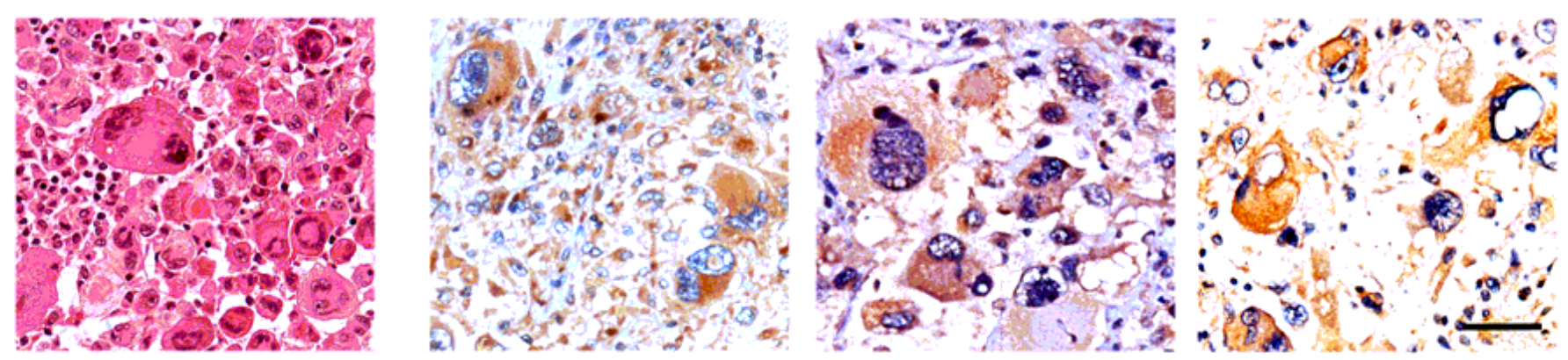

Figure 7

Appearance of germ cell-like cells in human glioma tissues. (A) Immunohistochemistry assays showing the expression and colocalization of the indicated proteins in lower-grade gliomas. (B) Immunohistochemistry assays showing the expression and colocalization of the indicated proteins in grade IV gliomas. Scale bar=50 $\mu \mathrm{m}$. 


\section{Supplementary Files}

This is a list of supplementary files associated with this preprint. Click to download.

- SupplementaryAppendix.docx 